\title{
Meeting report : Innovation in biology for the next generation!
}

\author{
Nam-Soo Kim \\ Received: 29 August 2010 / Accepted: 31 August 2011 / Published online: 20 October 2011 \\ (C) The Genetics Society of Korea and Springer 2011
}

The $66^{\text {th }}$ Annual Meeting of the Korean Association of Biological Sciences was held August 18-19 at Hallym University, Chuncheon, Korea. The theme of the meeting this year was "Innovation in Biology for the Next Generation." Two plenary lectures by Prof. J. Mark Cock (National Center for Scientific Research, University of Paris) and Prof. Kyungjin Kim (Seoul National University, Korea) were followed by symposia, including over 540 posters, sponsored by the participating societies: the Korean Society of Integrative Biology, the Korean Society of Limnology, the Ecological Society of Korea, the Korean Society of Biological Education, the Korean Society of Systematic Zoology, and the Genetics Society of Korea (GSK).

Prof. Cock's plenary lecture focused on genome evolution in the brown algae. His group recently published the whole genome sequence of the filamentous brown alga Ectocarpus siliculosus (Cock et al., 2010). He discussed several interesting genomic features of the Ectocarpus genome including the presence of an extended set of light-harvesting and pigment biosynthesis genes and new metabolic processes. One particularly interesting result from the Ectocarpus genome is that the membrane-localized receptor kinase gene families have evolved independently in brown algae, plants and animals, suggesting a strong link between the emergence of the kinase gene families and the evolution of multicellularity in each lineage. While the gametophytic life cycle in higher organisms is dependent on the sporophytic life cycle, in algae the two life cycles are independent. The genes expressed during the transition from sporophytic to gametophyte cycles in brown algae were different from those expressed during that transition in higher eukaryotes. Prof. Cock also presented evidence that the protein OUROBOROS is a master regulator of the gametophyte to sporophyte life cycle transition in Ectocarpus (Coelho et al.

Nam-Soo Kim, Ph.D $(\bowtie)$

Editor-in-Chief, Genes \& Genomics

Department of Molecular Bioscience, Institute of Bioscience and Biotechnology, Kangwon National University, Chuncheon 200-701, Korea

e-mail: kimnamsu@kangwon.ac.kr
2011).

The second plenary lecturer was Prof. Kyungjin Kim, a leading scientist in molecular and cellular biology whose group focuses on the human circadian clock machinery. The circadian rhythm, which is ubiquitous in eukaryotes, is one of the most well established biological rhythms, but understanding its details in different organisms presents an ongoing challenge. Prof. Kim presented findings from his group on two topics: molecular regulation of a core component of the circadian clockwork, CLOCK:BMAL1 (Shim et al., 2007; Lee et al., 2008), and control of the circadian glucocorticoid rhythm by the adrenal peripheral clock (Son et al., 2008). He explained the complex subject in plain language, so the audience from diverse biological disciplines could understand his amusing last slide on how the cattle farmer can know when it is lunch time without a clock.

The GSK had two symposia at the meeting, one focused on the genetics of bioenergy and the other on next generation sequencing. Biofuels are an alternative to fossil fuels for transportation, and research in the biofuel sector is booming due to government subsidies driven by rising oil prices and the limited supply of fossil fuel. There are two types of biofuels: bioethanol and biodiesel. Both are environmentally cleaner than fossil fuels in terms of carbon dioxide emission because they are derived from organic biomass such as living animals and plants or their byproducts. More than 23.6 million gallons of bioethanol and biodiesel were produced for the biofuel market worldwide in 2009, a market estimated at about US\$44.9 billion. The market is projected to grow to US\$112.5 billion by 2019 (http:/www.globe-net.com/articles/2010/march/31/clean-energy-trends-2010.aspx?sub=11). The topic of the second symposium, next generation sequencing, is one of the fastest changing fields in biology. Since the human genome sequence was deciphered, the list of organisms for which the whole genome has been sequenced continues to grow rapidly. One by-product of these genome projects is the invention of new sequencing techniques. Sequencing costs are dropping so dramatically that we will see the personal genome era in the near future. Next generation sequencing is a very appropriate symposium topic for scientists in genetics. 
In the symposium on bioenergy, four speakers presented their results and reviews of their fields. Prof. Sun-Hyung Kim (University of Seoul, Korea) discussed the current status of the use of Miscanthus and Phragmites for biomass. There are 15 species in the genus Miscanthus, perennial grasses that are native to subtropical and tropical regions of Africa and Asia, but can extend into temperate eastern Asia. Miscanthus can be easily grown, even in marginal soil, and provides a promising alternative to corn and other grains. One disadvantage of bioethanol production from grains like corn is negative effects on the food market, including instability and inflation in grain prices. The sterile triploid hybrid plant Miscanthus giganteus $(2 \mathrm{n}=3 \mathrm{x}=57)$ has attracted the interest of many plant biotechnologists because of its rapid growth, low mineral content, and high biomass. Prof. Kim introduced biotechnological modifications of $M$. giganteus that enhance bioethanol production. Because lignin poses a major obstacle in bioethanol production from plants, he is trying to modify the lignin composition and lignification process during plant development.

The second speaker was Dr. Yong-Hwa Lee (Rural Development Administration, Korea), who discussed the current status of rapeseed cultivar development for biodiesel production. The cost of converting rapeseed oil to biodiesel is low relative to other plant oils because the gelling temperature of rapeseed oil is low. In Korea, cultivation of rapeseed plants in rice paddies during the winter is of great interest for biodiesel because rice paddies are not in use at that time of year. In a first step towards that goal, Dr. Lee screened for genes expressed in cold-tolerant accessions to use as markers in a breeding program for cold-tolerant rapeseed cultivars.

The third speaker was Prof. Sung Ok Han (Korea University, Korea), a microbiologist developing designer microbes for biofuel production. The major obstacle in the biofuel industry is the high cost of converting biomass to biofuels. However, nature provides both simple and complex enzyme hydrolysis systems for degradation of biomass that can be used as guides. Han's group developed a complex enzyme system that significantly improves the hydrolysis of biomass materials. They constructed a minicellulosome consisting of a chimeric endoglucanase (cCelE) from Clostridium thermocellium and a chimeric agarase (cAgaB) from Zobellia galactanivorans fused with a dockerin domain from $C$. cellulovorans EngB. When expressed in Corynebacterium glutamicum, this minicellulosome plus an agarolytic complex from Escherichia coli increased activity against carboxymethyl cellulose or an agar substrate by 2.8 -fold and 1.4-fold, respectively, compared with that for the corresponding enzyme alone. Moreover, a Sacchromyces cereviceae strain expressing minicellulosome could ferment amorphous cellulose into ethanol with the aid of $\beta$-glucanase 1 produced from Saccharomycopsis fibuligera. In fermentation tests with $10 \mathrm{gL}^{-1}$ initial carboxymethyl cellulose, $3.45 \mathrm{gL}^{-1}$ ethanol was produced after 16 hours. Thus, the development of enzyme complexes with synergistic activity may lead to the commercial production of useful products from biomass materials with cost effectiveness.

The fourth speaker was Prof. Jae-Heung Ko (Kyung-Hee University, Korea) who spoke about biotechnological improvement of lignocellulosic feedstock for enhanced biofuel production. His main interest is on the secondary cell wall as a source of primary sugars for liquid biofuel production. The secondary cell walls are the most abundant biomass on earth, composed of a complex mixture of the structural polymers cellulose, hemicelluloses, and lignin. These polymers are very recalcitrant to enzymatic digestion, which presents challenges for bioconversion of lignocellulosic biomass. Prof. Ko's group has made great progress in understanding the transcriptional regulatory network that controls secondary wall biosynthesis. Prof. Ko provided an update on his group's achievements in experimental approaches to developing xylem-specific promoters for altering regulation.

The second GSK symposium topic was next generation sequencing (NGS). The first speaker was Dr. Ik-Young Choi (NICEM, Seoul National University, Korea) who summarized the history of the development of sequencing technology for the diverse audience in attendance. Since the classical and laborious methods developed by Gilbert and Maxam as well as Sanger and coworkers, several major technical breakthroughs have revolutionized nucleotide sequencing. These techniques include capillary electrophoresis, dye-termination, and polymerase chain reaction amplification. Dr. Choi compared the efficiencies of current techniques such as 454 pyrosequencing, Illumina (Solexa) sequencing, Ion semiconductor sequencing, and DNA nanoball sequencing. He also mentioned how NGS techniques can be applied in the analyses of complex biological phenomena including epigenetics, exom analysis, and gene regulation. He predicted that the fast development of sequencing technologies will bring personal genome projects to the forefront in the near future.

The second speaker was Dr. Namshin Kim (Korean Bioinformation Center, Korea Research institute of Bioscience and Biotechnology, Korea). Dr. Kim discussed the bioinformatics of NGS from disease through de novo assembly. He described a bioinformatic approach that he is using to identify the genetic basis of rare human diseases through the analysis of loss-of-function mutations caused by single nucleotide polymorphisms or In/Dels. Dr. Kim explained that current re-sequencing projects of Korean individuals using published reference human sequences have a critical need for development of new algorithms and software tools for predicting driver mutations for rare diseases, and for development of other bioinformatic tools to assemble and process the massive datasets. Dr. Kim also briefly introduced the pig genome sequencing project.

The third speaker was Prof. Sun-Min Ahn (Gachon University of Medicine, Korea) who discussed diploidomics, 
which he views as a next phase in human genome re-sequencing. He pointed out the necessity of our understanding the fundamental importance of diploidy in human genomes. Prof. Ahn emphasized the importance of haplotype information in human genome re-sequencing, and that it can be difficult to determine haplotype information because of the technical limitations of allelic phasing. To explain diploidomics, Prof. Ahn presented real pedigree data showing compound heterozygosity of a rare disease, and discussed how his approaches to compound heterozygosity analysis might help to understand human diseases associated with haploinsufficiency.

The last speaker was Prof. Chanseok Shin (Seoul National University, Korea) who gave an update on the regulation of gene expression by cellular microRNAs. The presence of non-coding RNAs like microRNAs is one of many unexpected finding from eukaryotic genome projects. Regulation by microRNAs depends on base pairing between microRNA and target mRNA at the "centered sites." In plants, the microRNA and target mRNA form almost perfect base pairing, which induces formation of the RNA-induced silencing complex, cleavage of target mRNA between the $10^{\text {th }}$ and $11^{\text {th }}$ nucleotides, and subsequent degradation of the target mRNA. In contrast, in animals, microRNAs form base pairing between the $2^{\text {nd }}$ and $7^{\text {th }}$ nucleotides with the target mRNA forming a so-called "8-mer seed-matched site," which results in non-cleavage mediated instability of the target mRNA. Prof. Shin presented some evidence from omics analyses that animal mRNAs can also be regulated by microRNAs via a cleavage mechanism.
The two symposia organized by the GSK attracted many scientists and graduate students, including scientists from other societies as well as GSK members. I can attest that both symposiums were highly successful.

\section{References}

Cock JM, Sterk L, Rouze P, Scornet D, Allen AE, Amoutzias G, Anthouard V, Artiguenave F, Aury JM, Badger JH et al. 2010. The Ectocarpus genome and the independent evolution of multicellularity in brown algae. Nature 465: 617-621.

Coelho SM, Godfroy O, Arun A, Le Corguille G, Peters AF, and Cock JM. 2011. OUROBOROS is a master regulator of the gametophyte to sporophyte life cycle transition in the brown alga Ectocarpus. Proc. Natl. Acad. Sci. USA doi: 10.1073-PNAS. 1102274108.

Lee J, Lee Y, Lee MJ, Park E, Kang SH, Chung CH, Lee KH, and Kim K. 2008. Dual modification of BMAL1 by SUMO2/3 and ubiquitin promotes circadian activation of the CLOCK/BMAL1 complex. Mol. Cell. Biol. 28: 6056-6065.

Shim HS, Kim H, Lee J, Son GH, Cho S, Kang SH, Seen DS, Lee $\mathrm{KH}$ and Kim K. 2007. Rapid activation of CLOCK by $\mathrm{Ca}^{2+}$-dependent kinase $\mathrm{C}$ mediates resetting of mammalian circadian clock. EMBO rep. 8:366-371.

Son GH, Chung S, Choe HK, Kim DH, Baik SM, Lee H, Lee HW, Choi S, Sun W, Kim H et al. 2008. Adrenal peripheral clock controls the autonomous circadian rhythm of glucocorticoid by causing rhythmic steroid production. Proc. Natl. Acad. Sci. USA 105: 20970-20975. 